\title{
Transatlantica
}

Revue d'études américaines. American Studies Journal

\section{Postface. Postérités du cinéma classique hollywoodien dans les remontages expérimentaux contemporains}

Marie-Pierre Burquier

\section{(2) OpenEdition}

Journals

Édition électronique

URL : https://journals.openedition.org/transatlantica/14276

DOI : 10.4000/transatlantica. 14276

ISSN : 1765-2766

Éditeur

Association française d'Etudes Américaines (AFEA)

Référence électronique

Marie-Pierre Burquier, «Postface. Postérités du cinéma classique hollywoodien dans les remontages expérimentaux contemporains », Transatlantica [En ligne], 1 | 2019, mis en ligne le 01 juillet 2020,

consulté le 01 février 2023. URL : http://journals.openedition.org/transatlantica/14276 ; DOI : https:// doi.org/10.4000/transatlantica. 14276

Ce document a été généré automatiquement le 1 février 2023.

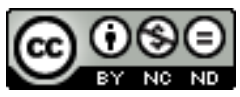

Creative Commons - Attribution - Pas d'Utilisation Commerciale - Pas de Modification 4.0 International - CC BY-NC-ND 4.0

https://creativecommons.org/licenses/by-nc-nd/4.0/ 


\title{
Postface. Postérités du cinéma classique hollywoodien dans les remontages expérimentaux contemporains
}

\author{
Marie-Pierre Burquier
}

Plus une œuvre est de haute qualité, plus elle reste, même dans le plus fugitif contact avec son sens, susceptible d'être encore traduite. Walter Benjamin

\section{Le cinéma classique hollywoodien : un cinéma du revisionnage, des relectures, et du remploi}

1 Connu pour reposer sur un mode de fabrication en série, avec des systèmes de reprises, de répétitions, de variations ou de recyclage ${ }^{1}$, le cinéma classique hollywoodien semble également se prêter tout particulièrement aux pratiques de revisionnages et de relectures. Cette tendance à revoir ou à réviser ce cinéma populaire s'explique d'abord par sa densité sans égal, par sa plénitude (Moure 15), ainsi que par l'épaisseur sémantique de ses récits. Les films issus de ce système de production ${ }^{2}$ semblent pour ces raisons ne cesser de s'enrichir au fil de leur redécouverte, que ce soit par le simple fait de les regarder à nouveau, par le renouvellement de leur analyse selon le contexte de réception, ou par des pratiques appropriationnistes, autant de moyens qui permettent de revisiter en profondeur ces œuvres qui ont marqué durablement l'histoire du cinéma.

2 La richesse de l'Hollywood classique n'est plus à démontrer; de nombreuses études se sont accordées sur l'ordonnancement de ses mises-en-scène, sur sa perfection formelle, sur son style homogène, et sur sa lisibilité quasi-universelle. Malgré son apparente transparence ${ }^{3}$, le contenu de cette iconographie demeure toutefois fortement régulé ou 
limité. Tous les sujets moralement discutables pour l'époque sont en effet évacués des écrans dès l'institution officielle du Code de censure, plus connu sous le nom du Code Hays, adopté en 1930 et dont la mise en œuvre se renforce à partir de 1934. Un paradoxe semble alors traverser le cinéma classique hollywoodien : tout en cherchant à se présenter comme pur reflet de la réalité, il travaille à évacuer les traces d'un réel indésirable, ou les signes de non conformité à une norme tacitement établie. Visuellement, cela est traduit par des images lisses, intactes, lustrées, sans flottement ni incertitude ; les ombres involontaires sont chassées du cadre, tout comme les sautes dans le montage, les moments de silence, de vide ou de suspension de la narration. Tout ce qui pourrait être l'occasion d'un investissement personnel de l'intrigue, comme les moments de creux, les ellipses ou une forte présence du hors-champ (Nacache), est évacué afin de pouvoir anticiper ou réguler le mieux possible les réceptions du spectateur, pour des raisons aussi bien morales qu'économiques (les films doivent chacun attirer en salle le plus large public possible).

3 Si plusieurs études relèvent une croyance et une adhésion généralisée à cet univers de substitution familier, "agréable » et «rassurant $»^{4}$ (Bidaud 251), l'effondrement du Code Hays à la fin des années 50 et la libération des représentations qui s'ensuit conduit à un nouveau rapport aux images classiques hollywoodiennes. À partir des années 70, des formes de suspicion émergent suite à la prise de conscience non seulement des processus de sélection qui traversent les récits, mais également du façonnement idéologique que certaines fictions contribuent à renforcer. Dans son article «Visual Pleasure and Narrative Cinema ", publié pour la première fois en 1975, la théoricienne britannique Laura Mulvey s'attache à montrer que ces récits reconduisent des schémas patriarcaux et dissimulent de puissants instruments de domination sociale et sexuelle. Selon elle, ce cinéma est surtout orienté vers la satisfaction du plaisir voyeuriste du spectateur masculin, et ne laisse pas de place pour l'avènement d'autres regards face aux images appréhendées ${ }^{5}$. Elle dénonce également l'hypocrisie d'un cinéma largement régulé par la censure mais demeurant plus ou moins permissif vis-à-vis de la représentation du corps féminin, puisqu'il expose régulièrement des corps réduits à l'état de «spectacle ", présents principalement pour être regardés et souvent à la merci des instances masculines qui cadrent le récit.

Bien que Laura Mulvey soit par la suite revenue sur cette lecture plusieurs fois jugée restrictive ${ }^{6}$, sa volonté de réinvestir ces images à l'aide des études féministes et des gender studies a eu pour conséquence l'émergence d'un ensemble de lectures proposant une alternative à cette iconographie dogmatique et cherchant à se dégager des regards préconçus qu'elle convoque. Il est par ailleurs important de rappeler que le code de censure n'a pas seulement chassé un ensemble de discours subversifs, mais a surtout conduit au développement de stratégies d'autorégulation de la part des réalisateurs hollywoodiens qui cherchent à anticiper d'éventuels rejets ou des coupes arbitraires, et excellent souvent dans l'art du sous-entendu. Cette forme d'autocensure contribue à l'avènement typiquement hollywoodien de tactiques de contournement ainsi que de sous-textes subversifs qui se manifestent par exemple à travers l'établissement de dialogues à double entente, d'effets de montage, d'organisation du hors-champ, etc. À travers l'usage de l'allusion, de l'ellipse, de gestes ou de regards connotés, ces réalisateurs sont en effet parvenus à laisser transparaitre des sens jugés alors plus ou moins « immoraux » au sein des films (à la condition d'un spectateur réceptif) tout en échappant à l'attention des censeurs. Le revisionnement critique initié par Laura Mulvey permet aussi de revenir, dans un contexte contemporain, à cet art du décodage 
des images hollywoodiennes, afin d'en saisir toute l'épaisseur et l'ambiguïté constitutive.

5 Considérer l'après du cinéma classique hollywoodien, voir "ce qu'il en reste " aujourd'hui, comme s'y attache ce dossier d'étude sur la postérité d'Autant en emporte le vent, doit alors nécessairement prendre en compte l'évolution de ces regards. Une mutation profonde est d'ailleurs observée plus nettement à partir des années 1980-90 avec ce qui a été désigné comme le Hollywood Turn ${ }^{7}$. Ce «tournant» est d'abord lié aux mutations économiques du cinéma hollywoodien dans son ensemble (et non pas exclusivement sa période dite classique), liées au développement des blockbusters et à l'avènement récent de nouvelles plateformes de visionnage qui bouleversent ses modes de consommation. Cette mutation est également liée à l'émergence, certes plus discrète, d'un ensemble de remontages artistiques, dans la tradition du cinéma expérimental et de l'art vidéo, qui prennent massivement pour cible les films classiques hollywoodiens à partir des années $1990^{\circ}$. Ces travaux tentent de dégager de nouvelles manières d'appréhender ces images populaires, le plus souvent à l'aide des technologies récentes de l'image et de l'informatique, ce qui génère de nouveaux modes de visionnements. En cherchant à voir ce qui peut encore en être dit, ils poursuivent par leur pratique personnelle l'écriture de ce cinéma-monument. À travers des gestes souvent minimaux, tels que la répétition, la boucle, le bégaiement, qui acquièrent entre leurs mains une portée révélatrice, ces artistes-vidéastes développent une nouvelle herméneutique contemporaine des images classiques hollywoodiennes. En modifiant leur rythme, leur orientation, leur montage, ils font en effet apparaitre les différents sous-textes qui les traversent et les confrontent ainsi à leur régime du refoulement et du manque.

6 Ces pratiques semblent directement résulter du revisionnage intensif des films hollywoodiens, voire de certaines scènes ou de certains motifs vus par ces artistes dès l'enfances, suivant un regard tout aussi nostalgique que critique et amusé (pouvant parfois être rapproché de la veine parodique). Ces modes de survivance des films s'inscrivent donc dans la lignée des différents renouveaux du film Autant en emporte le vent tour à tour évoqués dans ce dossier. Ils permettent eux aussi de poser un nouveau regard sur des films grand public, et appellent, de manière fortuite ou non, à la révision continuelle de l'histoire du cinéma classique hollywoodien. Ainsi déplacée dans le domaine de l'art vidéo et de l'art contemporain, l'iconographie classique hollywoodienne devient un matériau capable de questionner nos modes de représentation contemporains.

\section{La postérité des chefs-d'œuvre hollywoodiens dans le cinéma de remontage : l'absence d'Autant en emporte le vent}

7 Outre les remakes, les suites, les restaurations en chaîne, nombre de grands classiques hollywoodiens entrent par le biais de films de remontages dans la postérité, une postérité certes relative, puisque ces œuvres sont plus difficilement accessibles. Pour ne citer que quelques exemples, Eve de Joseph Mankiewicz a tour à tour été repris par les artistes Roger Jacoby (Aged in Wood, 1975), Jim Hubbard (Footsteps on the Ceiling, 2013) et Martin Arnold (Dissociated, 2002); Boulevard du crépuscule par l'Américain Gregg Biermann (Out there in the Dark, 2007); le King Kong de Merian C. Cooper et Ernest 
Shoedsack par l'Allemand Christoph Girardet (Release, 1996), ou encore Le train sifflera trois fois par Martin Arnold (Forsaken, 2002) et Gregg Biermann (High Noon Reflection, 2009). Il peut alors être surprenant de ne pas voir figurer parmi ces reprises la grande fresque hollywoodienne d'Autant en emporte le vent. S'il a bien été l'objet d'innombrables citations, de parodies, de nombreuses restaurations ${ }^{10}$, et s'il existe une communauté d'œuvres cinématographiques déterminées en partie par l'œuvre de Margaret Mitchell ou l'adaptation de Victor Fleming, le film n'a jamais donné lieu à une suite ou un remake en bonne et due forme, ni, non plus, à un remontage expérimental ou des essais vidéo de fanfiction notables (une forme de prolongement fréquente des grands classiques hollywoodiens, fondée elle aussi sur l'appropriation personnelle des images filmiques). Il est d'autant plus curieux qu'Autant en emporte le vent reste pour l'instant inemployé par ces artistes-vidéastes alors qu'il semble offrir une matière première riche ne serait-ce que par sa durée. Ses images, qui ont marqué durablement l'histoire du cinéma, sa matière graphique singulière (avec ses teintes orangées et ocre, ses fonds en toiles peintes, ses ombres découpées) semblent en effet appeler directement au traitement plastique ou au remodelage.

Par comparaison, Le Magicien d'Oz, sorti la même année et dont deux des réalisateurs (Fleming et Cukor) ont aussi participé à la production de Selznick, est encore aujourd'hui l'objet de nombreuses appropriations. Il est alors possible de penser, avec Sarah Hatchuel, qui note dans son article pour ce dossier de Transatlantica que le film est souvent pris « comme un aboutissement ultime », qu'Autant en emporte le vent a été délaissé jusqu'ici par les artistes contemporains parce que l'œuvre est d'une plénitude telle qu'elle semble impossible à réviser. Toutefois, cette absence semble surtout pouvoir être expliquée, comme le note Pierre Berthomieu dans l'entretien vidéo figurant dans ce dossier, par des raisons idéologiques évidentes. Sa vision idéalisée du Sud, sa sentimentalisation de l'esclavagisme et sa représentation des minorités raciales en font une œuvre particulièrement délicate à revisiter bien qu'il semble aussi, et tout autant, nécessaire de le faire. Cette réticence à reprendre ou même à revoir le film aujourd'hui s'illustre d'ailleurs parfaitement dans le soudain retrait de l'œuvre de la plateforme de streaming HBO Max. Rappelant les raisons qui viennent d'être mentionnées, le scénariste John Ridley déclare, dans une tribune publiée le 8 juin 2020 dans le Los Angeles Times, en plein mouvement de protestation contre les violences policières racistes aux États-Unis, qu'il est inacceptable qu'Autant en emporte le vent soit aisément accessible en ligne sans contenu qui viendrait l'encadrer et en expliciterait les préjugés idéologiques. La plateforme prévoit la remise en ligne du film prochainement, accompagné cette fois-ci d'une notice historique et critique. Cette querelle, toutefois, témoigne bien des difficultés qui entoureraient la reprise d'une telle œuvre dans une logique appropriationniste; elle constitue une explication possible de sa postérité limitée, voire inexistante, dans le cinéma expérimental et l'art vidéo contemporain.

Paradoxalement, pourtant, les pratiques de remontage sont l'outil idéal pour susciter des regards renouvelés sur les œuvres du passé et pourraient parfaitement fournir le bagage critique nécessaire au mode de présentation du film aujourd'hui. Plutôt que de chercher à "supprimer » Autant en emporte le vent, de telles propositions artistiques pourraient en effet être un moyen de conduire à de nouvelles manières de « retourner à Tara » et de questionner à la fois le devenir matériel et idéologique du film.

10 Les œuvres de remontage qui s'intéressent aux représentations problématiques des minorités raciales dans les fictions classiques hollywoodiennes sont encore très 
ponctuelles et ne constituent pas un corpus dense et unifié ${ }^{11}$. Toutefois, un large ensemble d'œuvres s'attache à déconstruire les représentations des minorités de genre $^{12}$ (Wees 15), sans doute plus "présentes » que les minorités raciales dans les images des années cinquante. Ces travaux de remploi prennent souvent pour matériau des films qui proposent déjà une alternative aux modèles classiques d'identification. Avec ses personnages anticonformistes, sa distance avec les conventions ou les standards genrés de la fin des années 1930, Autant en emporte le vent pourrait présenter un matériau idéal à de semblables mises au jour. Comme le montre Emmeline Gros dans son article "Seeing Margaret Mitchell's Gone With the Wind with Fresh Eyes ", Autant en emporte le vent offre en effet une matière riche pour penser la représentation du genre et de la sexualité. Il propose aux spectateurs de la fin des années 1930 des modèles inédits d'identification, notamment par le biais de Scarlett O'Hara qui agit régulièrement à contre-courant des normes. Scarlett correspond d'ailleurs parfaitement aux figures de prédilection des artistes de remontage : des personnages de femmes fortes et indépendantes, qui autorisent souvent le développement d'un autre regard au sein des fictions. Reviennent par exemple régulièrement dans leurs travaux Judy Garland, Bette Davis dans Eve, ou Marlene Dietrich et Greta Garbo dans leurs rôles à la sexualité ambiguë. En s'intéressant à ces personnages féminins nonconventionnels, les artistes de remontage parviennent à montrer que les récits classiques ne s'apparentent pas seulement à des outils de façonnage idéologique mais peuvent contenir en leur sein un espace possible pour des formes d'émancipation. En actualisant ${ }^{13}$ ces images du passé, ces films invitent alors à la poursuite de l'écriture de la mythologie cinématographique américaine, à travers la mise au jour de ses images manquantes, de ses histoires non encore écrites, tout en convoquant l'ensemble de ses futurs encore à venir.

\section{Revisiter l'archive hollywoodienne : vers une nouvelle historiographie du cinéma classique hollywoodien? Le cas du Magicien d'Oz}

11 J'aimerais pour finir évoquer brièvement trois œuvres réalisées à partir du film $L e$ Magicien d'Oz de 1939 afin de donner une idée concrète des différentes survivances artistiques d'un chef-d'œuvre emblématique du cinéma hollywoodien. Dans son courtmétrage Utopia Variation (2008), l'artiste américain Gregg Biermann reprend les premiers plans chantés du film, lors desquels Judy Garland (incarnant Dorothy Gale) rêvasse près d'une roulotte. Ici, ce plan initial est démultiplié grâce à un algorithme numérique jusqu'à former un damier de vingt-quatre images semblables au sein du cadre, chacune décalées de quelques secondes dans le temps. 
Fig. 1 Utopia Variation, Gregg Biermann, 2008

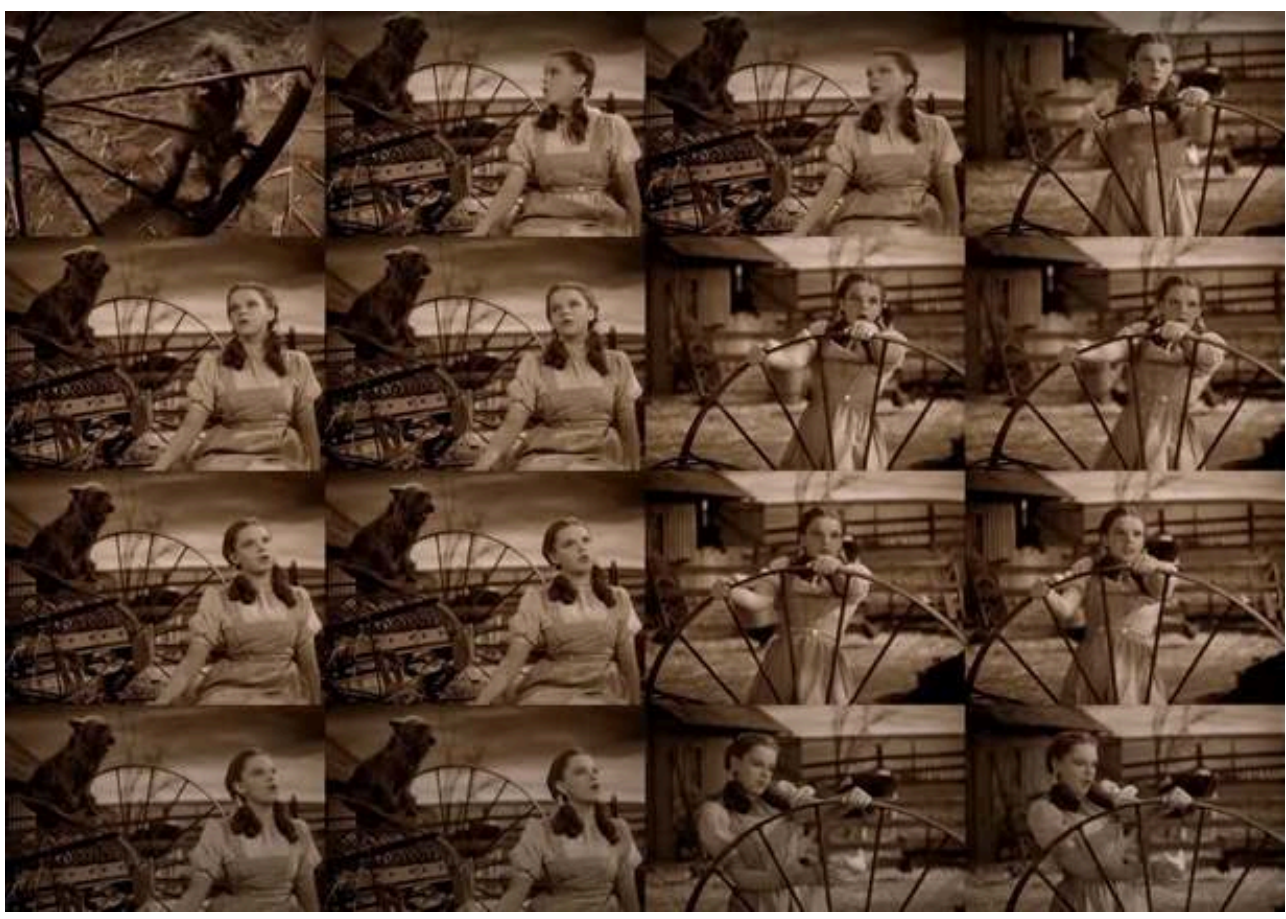

Crédits : Courtesy of the artist, Gregg Biermann.

12 Ce réagencement donne l'impression d'une prolifération autonome de l'image liée à un bug, entraînant une saturation visuelle et un chant polyphonique aux paroles désormais indiscernables. Par cette démultiplication ad nauseam d'une même séquence, Gregg Biermann s'amuse des dérives possiblement monstrueuses des modes de visionnement liés aux nouveaux outils digitaux. Il souligne également un rapport défaillant aux images du passé et leur nécessaire réadaptation à notre nouvelle « frénésie du visible » (Beugnet 103).

Le court-métrage du collectif australien SODA_JERK ${ }^{14}$, After the Rainbow (2009), questionne également le devenir de l'icône Garland à partir du personnage de Dorothy. Le film débute par la reprise d'une des premières séquences, lorsque la fillette, après avoir quitté le foyer familial, est menacée par l'arrivée d'une tornade. Les images sont alors retravaillées de l'intérieur : dès l'arrivée de la tempête à l'arrière-plan (élément déclencheur du voyage de Dorothy dans l'autre monde), le fond de l'image commence à crépiter et à brûler partiellement, comme si l'événement météorologique se déportait directement sur la bande du film ${ }^{15}$. 
Fig. 2 After the Rainbow, SODA_JERK, 2009

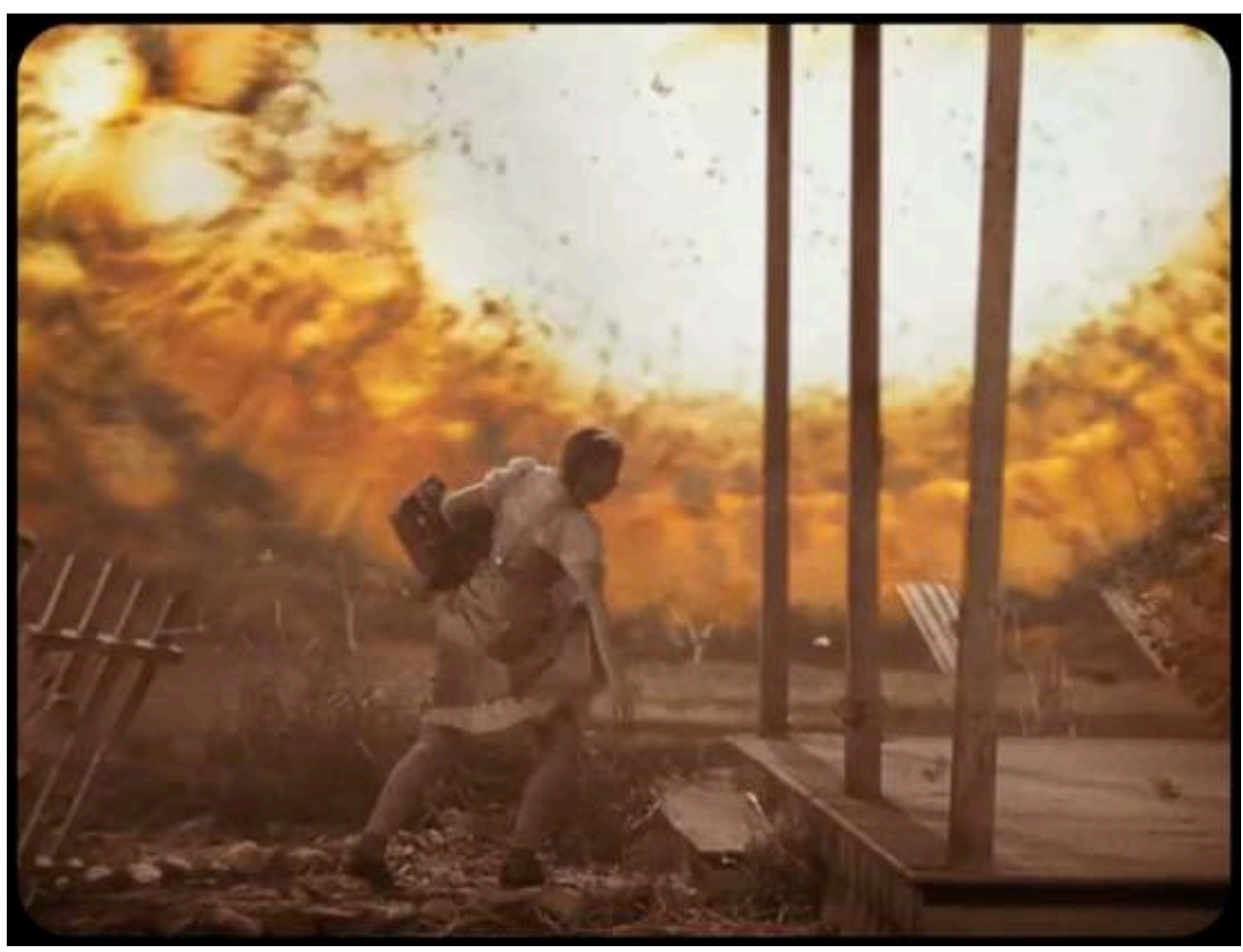

Crédits : Courtesy of the artist, SODA_JERK.

14 L'entrée de Dorothy dans l'autre monde devient ici un accès à une temporalité future, ces altérations soulignant le passage accéléré du temps en prenant l'aspect de vieilles pellicules détériorées. D'ailleurs, suite à la tornade, Dorothy est bien propulsée dans ce qui semble être sa destinée : par des effets d'incrustation, les images du Magicien d'Oz sont associées à un numéro chanté de Judy Garland alors en fin de carrière ${ }^{16}$. Dans cette séquence combinatoire, Judy Garland adulte tourne le dos à sa jeunesse, représentée par Dorothy, avant d'être avalée par un fond noir. Cette condensation d'images de la star à différents âges permet de revenir sur la carrière désenchantée et fulgurante de l'actrice ${ }^{17}$. After the Rainbow rappelle ici les dérives du star system en soulignant la menace omniprésente du vieillissement dans l'industrie hollywoodienne.

Dans son installation photographique Fleming's The Wizard of $O z, 2001$, issue de sa série "Illuminated Average Series", l'artiste Jim Campbell joue, lui aussi, sur la superposition des temps à partir du film de Fleming. Son œuvre consiste en une seule image fixe qui superpose, sous la forme de calques transparents, l'ensemble des plans du film, désormais méconnaissables. 
Fig. 3 Illuminated Average \#5, Fleming's The Wizard of Oz, Jim CAMPBELL, 2001 (24 x 18 inches)

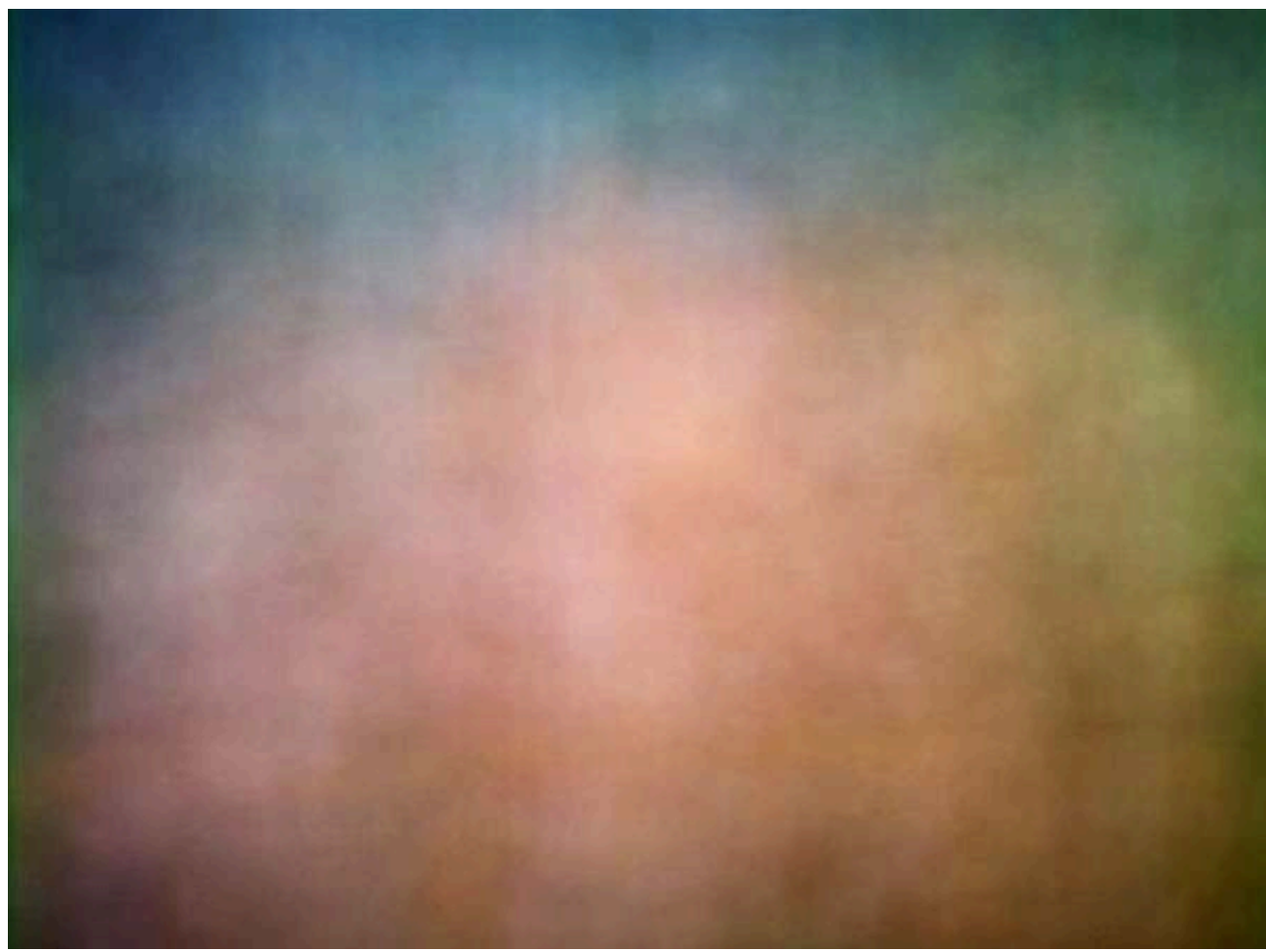

Averaged over 1 hour, 42 minutes (entire film)

Edition of three $24 \times 18$ inches, Duratrans, lightbox

Crédits : Courtesy of the artist Jim Campbell.

Cette image-palimpseste est réalisée à l'aide d'un algorithme numérique qui permet de condenser le film en un seul «plan » qui les comprend tous. L'œuvre finale laisse voir différents aplats de couleurs, une masse rougeoyante entourée d'aplats vert, bleu, jaune, renvoyant tour à tour aux ruby slippers portées par Dorothy, à la Yellow-Brick Road empruntée par les personnages, et à l'ensemble de l'univers graphique du film. Malgré un sentiment de déperdition ou d'écrasement lié à la compression algorithmique, cette image soluble, floue, offre au contraire une simultanéité inédite du film, qui peut alors être appréhendé dans sa totalité d'un seul regard englobant. Pour reprendre le vocabulaire de Gilles Deleuze, cette œuvre est une parfaite actualisation d'une image-mentale ${ }^{18}$, d'une image rêvée par nous et rendue manifeste par un logiciel, qui convoque, en une masse abstraite, l'ensemble des variations du film originel, tout en en renouvelant l'aspect plastique.

Ces trois devenirs possibles du Magicien d'Oz permettent d'imaginer ce qu'auraient pu ou ce que pourraient être de telles appropriations d'Autant en emporte le vent. Chacun s'intéresse à la postérité technologique du film, à sa renaissance matérielle, tout en permettant de mieux comprendre son esthétique originelle et de renseigner son fonctionnement. Ces œuvres font ressurgir, de manière programmatique ou non, soit des sens et des modes de visionnement inédits (comme c'est le cas dans les trois films analysés), soit l'impensé idéologique des représentations originelles (dans d'autres œuvres appartenant à ce large corpus de remontage des films hollywoodiens). Ce renouvellement concerne également les modes de diffusion puisque ces œuvres ne sont pas projetées dans les salles traditionnelles de cinéma mais sur des moniteurs ou des écrans situés dans des musées ou galeries. Ces travaux montrent qu'il est alors 
nécessaire de revisiter l'archive hollywoodienne en prenant en compte la façon dont ces transcodages et cette nouvelle transmédialité dessinent un espace commun à l'art plastique et au cinéma.

\section{BIBLIOGRAPHIE}

BEUGNET, Martine. L'attrait du flou. Crisnée : Yellow Now, 2017.

BEUGNET, Martine et Jane Sillars. « The Reflexive Turn: Mediating and Remediating Hollywood: Introduction ». Screen, vol. 58, no. 2, 2017, p. 197-201.

BENJAMIN, Walter. "La tâche du traducteur ». 1923. Euvres 1. Traduit de l'allemand par M. de Gandillac, R. Rochlitz et P. Rusch. Paris : Gallimard, coll. Folio essais, 2007, p. 260-261.

BIDAUD, Anne-Marie. « Le cinéma américain ou les ambiguïtés idéologiques d'un médium de masse ». Revue Française d'Études Américaines, no. 6, 1978, numéro thématique : « Mass media et idéologie aux Etats-Unis », p. 251-268.

MOURE, José. Vers une esthétique du vide au cinéma. Paris : L'Harmattan, 1997.

MULVEY, Laura. « Visual Pleasure and Narrative Cinema ». Screen, vol. 16, no. 3, 1975, p. 6-18.

NACACHE, Jacqueline. Hollywood, l'ellipse et l'infilmé. Paris : L'Harmattan, 2001.

RIDLEY, John. “Op-Ed: Hey, HBO, 'Gone With the Wind' romanticizes the horrors of slavery. Take it off your platform for now.” Los Angeles Times. 8 juin 2020. https://www.latimes.com/opinion/ story/2020-06-08/hbo-max-racism-gone-with-the-wind-movie. Page consultée le 10 juin 2020.

WEES, William C. « The Ambiguous Aura of Hollywood Stars in Avant-Garde Found-Footage Film ». Cinema Journal, vol. 41, no. 2, 2002, p. 3-18.

Filmographie et œuvres d'art

After the Rainbow. Réal. SODA_JERK, 2009.

Aged in Wood. Réal. Roger Jacoby, 1975.

All About Eve (Ève). Réal. Joseph L. Mankiewicz. Scénario de Joseph L. Mankiewicz d'après The Wisdom of Eve de Mary Orr. Avec Bette Davis (Margo Channing), Anne Baxter (Eve Harrington), George Sanders (Addison DeWitt), Celeste Holm (Karen Richards), Gary Merrill (Vill Sampson). Twentieth Century Fox, 1950.

Dissociated. Réal. Martin Arnold, 2002.

Fleming's The Wizard of Oz. Réal. Jim Campbell, 2001. (24 x 18 inches). Plus d'1 heure, 42 minutes (film entier). Edition de trois $24 \times 18$ inches, Duratrans, lightbox.

Footsteps on the Ceiling. Réal. Jim Hubbard, 2013.

Forsaken. Réal. Martin Arnold, 2002.

Gone with the Wind (Autant en Emporte le Vent). Réal. Victor Fleming, Sam Wood, George Cukor. Scénario de Sidney Howard, d'après le roman de Margaret Mitchell. Avec Clark Gable (Rhett Butler), Leslie Howard (Ashley Wilkes), Vivien Leigh (Scarlett O'Hara), Olivia De Havilland 
(Melanie Hamilton), Hattie McDaniel (Mamma). Selznick International Pictures / MetroGoldwyn-Mayer, 1939.

High Noon (Le train sifflera trois fois). Réal. Fred Zinnemann. Scénario de Carl Foreman, d'après la nouvelle de John W. Cunningham. Avec Gary Cooper (Will Kane), Grace Kelly (Amy Fowler Kane), Lloyd Bridges (Harvey Pell). Stanley Kramer Productions, 1951.

High Noon Reflection. Réal. Gregg Biermann, 2009.

Invisible Ghost. Réal. Joseph H. Lewis. Scénario de Al Martin et Helen Martin. Avec Bela Lugosi (Dr. Charles Kessler), Polly Ann Young (Virginia Kessler), John McGuire (Ralph Dickson / Paul Dickson), Clarence Muse (Evans the Butler). Sam Katzman, 1941.

King Kong. Réal. Merian C. Cooper et Ernest B. Shoedsack. Scénario de James A. Creelman et Ruth Rose. Avec Fay Wray (Ann Darrow), Robert Armstrong (Carl Denham), Bruce Cabot (Jack Driscoll). RKO Pictures, 1933.

Lip. Réal. Tracey Moffatt, Gary Hillberg, 1999.

Meeting of Two Queens. Réal. Cecilia Barriga, 1991.

Out there in the Dark. Réal. Gregg Biermann, 2007.

Release. Réal. Christoph Girardet 1996.

Remembrance. Réal. Jerry Tartaglia, 1990.

Rock Hudson's Home Movies. Réal. Mark Rappaport, 1992.

Rose Hobart. Réal. Joseph Cornell, 1936.

A Star is Born. (Une Étoile est née). Réal. George Cukor. Scénario de Moss Hart. D’après le scénario de A star is born de Dorothy Parker, Alan Campbell, Robert Carson, William A. Wellman. Avec Judy Garland (Esther Blodgett / Vicki Lester), James Mason (Norman Maine), Jack Carson (Matt Libby). Warner Bros Pictures, 1953.

Sunset Boulevard (Boulevard du crépuscule). Réal. Billy Wilder. Scénario de Billy Wilder, Charles Brackett, D.M. Marshman Jr.. Avec William Holden (Joe Gillis), Gloria Swanson (Norma Desmond), Erich von Stroheim (lui-même), Nancy Olson (Betty Schefer). Prod. Charles Brackett, 1950.

Utopia Variation. Réal. Gregg Biermann, 2008.

The Wizard of $\mathrm{Oz}$ (Le Magicien d'Oz). Réal. Victor Fleming, King Vidor. Scénario Noel Langley, Florence Ryerson, Edgar Allan Woolf, d'après le livre de L. Frank Baum. Avec Judy Garland (Dorothée Carter / Dorothy Gale), Frank Morgan (le Magicien d'Oz / le professeur Merveille / le coher / le garde / le portier), Ray Bolger (Hunk / l'Epouvantail), Bert Lahr (le Lion peureux). Metro-Goldwyn-Mayer, 1939.

\section{NOTES}

1. Le cinéma classique hollywoodien reprend fréquemment les mêmes motifs au sein des films et produit régulièrement des suites, des nouvelles versions, des réadaptations ou des remakes.

2. Surtout ceux réalisés entre les années trente et les années cinquante, Âge d'or du style classique hollywoodien.

3. La transparence classique consiste en la recherche d'une cohésion avec le réel, dans le but de l'adhésion immédiate du spectateur à ce qu'il voit; cette transparence reste toutefois très relative comme le rappelle Pierre Berthomieu dans l'entretien vidéo figurant dans ce dossier. 
4. Elle évoque le cinéma américain en général comme «[...] un univers de substitution plus agréable et rassurant, parce qu'il répond à leur besoin [les spectateurs] de cohésion et de sécurité en évacuant de la fragmentation psychique et sociale ».

5. Qu'il s'agisse de la caméra, du personnage masculin dans la fiction ou du spectateur lui-même.

6. Notamment en 1981, dans son article "Afterthoughts on 'Visual Pleasure and Narrative Cinema' inspired by King Vidor's Duel in the Sun (1946) », présenté à la conférence "Cinema and Psychoanalysis ", Center for Media Studies, SUNY, Buffalo et publié en 1981 également dans la revue Framework.

7. Ce tournant historiographique a fait l'objet d'un colloque international à l'Université ParisDiderot en 2015 : «The Hollywood Turn: Viewing American Cinema Anew » et d'une publication (Beugnet et Sillars).

8. Ces pratiques se développent dans les années 1990 grâce à l'avènement d'internet et de logiciels de montage peu coûteux qui permettent une nouvelle accessibilité aux images (le remontage d'images hollywoodiennes a toutefois été initié en 1936 par l'artiste américain Joseph Cornell avec son court-métrage Rose Hobart).

9. De nombreux films jouent d'ailleurs sur la simple répétition systématique des mêmes fragments d'images.

10. Voir, pour plus de précisions, l'entretien avec Pierre Berthomieu, ainsi que l'article de Taïna Tuhkunen sur Went With The Wind (1976), la reprise parodique du film dans The Carol Burnett Show, figurant tous deux dans ce dossier.

11. C'est le cas, par exemple, dans le film de Martin Arnold, Deanimated (2002) au cours duquel l'artiste efface progressivement l'ensemble des personnages du film Invisible Ghost de Joseph $\mathrm{H}$. Lewis (1941) mais laisse perdurer plus longuement que les autres le personnage d'Evans, le majordome noir. N'ayant qu'un rôle annexe dans le récit originel, il devient ici figure centrale du nouveau « récit ». Voir également le court-métrage Lip de Tracey Moffatt et Gary Hillberg (1999).

12. Les artistes de remontage ont œuvré dans les années 1990, un contexte influencé par les théories queer et l'activisme LGBTQ. Je renvoie ici plus particulièrement aux films Remembrance de Jerry Tartaglia (1990), Meeting of Two Queens de Cecilia Barriga (1991) et Rock Hudson's Home Movies de Mark Rappaport (1992).

13. Je prends actualisation ici dans le double sens d'une adaptation au temps présent et de l'accès au visible de sens qui existent à l'état virtuel.

14. Il s'agit du second film d'une série débutée en 2005 intitulée « The Dark Matter Series »; elle rassemble à ce jour trois installations vidéo reposant sur la reprise d'images mettant en scènes des stars hollywoodiennes du passé, donnant l'illusion que les personnages qu'elles ont incarnés se rencontrent à différents âges.

15. Comme si la bande-image prenait feu, mais le film a bien été réalisé numériquement.

16. Elle est en train d'interpréter « The Man That Got Away » lors du show « Judy, Frank \& Dean, once in a lifetime » de 1962, un titre qu'elle a déjà chanté quelques années auparavant dans le film A Star is Born (George Cukor 1954).

17. La carrière de Judy Garland témoigne particulièrement des dérives du star system hollywoodien.

18. C'est-à-dire des images qui n'existent pas visiblement, mais qui surgissent dans l'intellect ou la mémoire du spectateur. 


\section{AUTEUR}

MARIE-PIERRE BURQUIER

LARCA-UMR8225, Université de Paris 